\title{
Exact sum rules for vector channel at finite temperature and their application to lattice $Q C D$ analysis
}

\author{
Daisuke Satow ${ }^{1, a}$ and Philipp Gubler ${ }^{2}$ \\ ${ }^{1}$ Institut für Theoretische Physik, Johann Wolfgang Goethe-Universität, Max-von-Laue-Str. 1, D-60438 \\ Frankfurt am Main, Germany \\ ${ }^{2}$ Department of Physics and Institute of Physics and Applied Physics, Yonsei University, Seoul 120-749, \\ Korea
}

\begin{abstract}
We derive three exact sum rules for the spectral function of the electromagnetic current with zero spatial momentum at finite temperature. Possible applications of the three sum rules to lattice computations of the spectral function and transport coefficients are also discussed: We propose an ansatz for the spectral function that can be applied to all three sum rules and fit it to available lattice data of the Euclidean vector correlator above the critical temperature. As a result, we obtain estimates for both the electrical conductivity $\sigma$ and the second order transport coefficient $\tau_{J}$.
\end{abstract}

\section{Introduction and Summary}

Among the properties of hadronic matter at finite temperature, whose dynamics is described by quantum chromodynamics (QCD), the spectral function of the electromagnetic current plays an important role since it contains the full information on the dilepton/photon production rate [1], the electrical conductivity, and the modification of the spectral properties of vector mesons at finite temperature. All these quantities have been intensively studied in the context of heavy ion collisions. The spectral function has therefore naturally been investigated within many approaches, such as perturbative QCD [2], the AdS/CFT correspondence [3], model calculations [4], low-energy effective theory based on hadronic degrees of freedom [5, 6], sum rules [7-10], and lattice QCD [11-19], which have led to a large number of diverse results. Under such circumstances, it is useful to have exact constraints on the spectral function that all approaches should satisfy. Especially in lattice QCD, which can be directly applicable only for static quantities, it would be useful to have such constraints since the spectral function is a dynamical quantity and thus can not be computed directly. One goal of the present paper is to provide such constraints in the form of sum rules, and discuss their applications to lattice QCD analysis.

In the first part of the manuscript, we derive the three sum rules of Eqs. (9), (10), and (13), of which the second and third one are written down here for the first time. The third one is valid in the large $N_{c}$ limit while the other two are exact for general $N_{c}$. For this purpose, we make use of a method developed for the energy-momentum tensor channel in an earlier work by Romatschke and Son [20]. We emphasize that these sum rules are exact, and valid both in hadron and QGP phases, as

\footnotetext{
ae-mail: dsato@th.physik.uni-frankfurt.de
} 


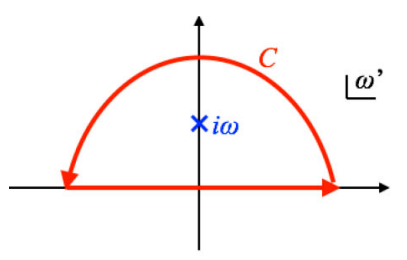

Figure 1. The contour $C$, used in the integral of Eq. (1).

long as hydrodynamics is reliable there. Next, we discuss potential applications of the sum rules to lattice QCD studies of the spectral function. These include the possibility of providing constraints to the spectral function ansatz used to fit the Euclidean vector correlator lattice data, improvements for this ansatz, and the extraction of the second order transport coefficient $\tau_{J}$ from the spectral function obtained from a fit to lattice data. More detailed analysis is in Ref. [21].

\section{Sum rules}

\subsection{Sum Rule 1}

The quantity we are interested in is the retarded Green function of the electromagnetic (EM) current: $G_{\mu \nu}^{R}(\omega, \mathbf{p}) \equiv i \int d t \int d^{3} \mathbf{x} e^{i \omega t-i \mathbf{p} \cdot \mathbf{x}} \theta(t)\left\langle\left[j^{\mu}(t, \mathbf{x}), j^{\nu}(0, \mathbf{0})\right]\right\rangle$, where $j^{\mu} \equiv e \sum_{f} q_{f} \bar{\psi}_{f} \gamma^{\mu} \psi_{f}$ is the EM current, and the average is taken over the thermal ensemble. Here $e$ is the electromagnetic coupling constant, $q_{f}$ the charge in each quark flavor, and $\psi_{f}$ the quark field with flavor $f$, respectively. At $|\mathbf{p}|=0$, there is only one independent component in the spatial components of this tensor, $G^{R}(\omega) \equiv G_{i i}^{R}(\omega, \mathbf{0}) / 3$, due to isotropy. In this paper, we limit ourselves to this case for simplicity.

First, to introduce the method developed in Ref. [20], we rederive the sum rule of Eq. (9), which has already been obtained in Ref. [15] from the current conservation law. The retarded Green function is known to be analytic in the upper half of the complex $\omega$ plane. This property enables us to derive various sum rules. Because of the residue theorem, we have

$$
\delta G^{R}(i \omega)-\delta G_{\infty}^{R}=\frac{1}{2 \pi i} \oint_{C} d \omega^{\prime} \frac{\delta G^{R}\left(\omega^{\prime}\right)-\delta G_{\infty}^{R}}{\omega^{\prime}-i \omega},
$$

for which the contour $C$ is shown in Fig. 1. Here $\delta$ stands for the subtraction of the $T=0$ value of $G^{R}(\omega), \delta G^{R}(\omega) \equiv G^{R}(\omega)-\left.G^{R}(\omega)\right|_{T=0}$. Due to this subtraction, the ultraviolet behavior of $G^{R}$ is improved so that the contribution from the arc with infinite radius becomes negligible. Another subtraction of $\left.\delta G_{\infty}^{R} \equiv \delta G^{R}(i \omega)\right|_{\omega \rightarrow \infty}$ is for removing any possibly remaining ultraviolet divergence. Taking the infinitesimal $\omega$ limit, we get

$$
\delta G^{R}(0)-\delta G_{\infty}^{R}=\frac{2}{\pi} \int_{0}^{\infty} \frac{d \omega}{\omega} \delta \rho(\omega),
$$

where we have made use of the fact that the real (imaginary) part of $G^{R}(\omega)$ is an even (odd) function of $\omega$, and introduced the spectral function, $\rho(\omega) \equiv \operatorname{Im} G^{R}(\omega)$. We also changed the integration variable to $\omega$ for simplicity.

On the left-hand side, the ultraviolet (UV) and infrared (IR) limits of $G^{R}$ constrain the spectral function integral through Eq. (2). The former quantity can be evaluated using the operator product expansion (OPE) $[22,23]$. Because of the subtraction of the $T=0$ piece, all terms with operators 
of mass dimensions less than four vanish, so that the asymptotic behavior at large $\omega$ is described by the operators with mass dimensions four. By computing the coefficients of such operators at leading order in $\alpha_{s}$, we get

$$
\delta G^{R}(\omega)=e^{2} \sum_{f} q_{f}^{2} \frac{1}{\omega^{2}}\left[2 m_{f} \delta\left\langle\bar{\psi}_{f} \psi_{f}\right\rangle+\frac{1}{12} \delta\left\langle\frac{\alpha_{s}}{\pi} G^{2}\right\rangle+\frac{8}{3} \delta\left\langle T_{f}^{00}\right\rangle\right]+O\left(\omega^{-4}\right)
$$

where $G_{a}^{\mu \nu} \equiv \partial^{\mu} A_{a}^{v}-\partial^{v} A_{a}^{\mu}-g f_{a b c} A_{b}^{\mu} A_{c}^{v}$ is the field strength, $G^{2} \equiv G_{\mu \nu}^{a} G^{a \mu \nu}, T_{f}^{\alpha \beta} \equiv i S \mathcal{S} \bar{\psi}_{f} \gamma^{\alpha} D^{\beta} \psi_{f}$ is the quark component to the traceless part of the energy-momentum tensor, $D^{\mu} \equiv \partial^{\mu}+i g A_{a}^{\mu} t^{a}$ the covariant derivative, $A_{a}^{\mu}$ the gluon field, $t^{a}$ the generator of the $S U\left(N_{c}\right)$ group in the fundamental representation, $f_{a b c}$ the structure constant of the $S U\left(N_{c}\right)$ group, $m_{f}$ the current quark mass, $g$ the QCD coupling constant, $\alpha_{s} \equiv g^{2} /(4 \pi)$, and $N_{c}$ the number of the colors. $S \mathcal{T}$ makes a tensor symmetric and traceless: $\mathcal{S T} O^{\alpha \beta} \equiv\left(O^{\alpha \beta}+O^{\beta \alpha}\right) / 2-g^{\alpha \beta} O_{\mu}^{\mu} / 4$. We note that having dropped higher order corrections to the coefficients above will be justified in the $\omega \rightarrow \infty$ limit, which allows us to use asymptotic freedom. Also note that the traceless gluonic component of the energy-momentum tensor $\left[T_{g}^{00}\right.$, defined above Eq. (4)] can also in principle appear in the OPE at finite temperature. We have dropped such a term since it vanishes at leading order in $\alpha_{s}$, but we will discuss below that it shows up once the operator mixing is taken into account. We retained the gluon condensate term though formally it is of higher order in $\alpha_{s}$, as it turns out to be finite even in the $\omega \rightarrow \infty$ limit due to its vanishing anomalous dimension. When considering the $\omega \rightarrow \infty$ limit, we need to take into account the effects of scaling and mixing of the operators, reflected in their anomalous dimensions. The anomalous dimensions of the chiral and gluon condensates are zero, so they do neither scale nor mix. On the other hand, the quark energy momentum tensor both scales and mixes with a respective gluonic operator. To understand this behavior, we rewrite the operator as $T_{f}^{00}=T_{f}^{\prime 00}+\left(T^{00}+2 \tilde{T}^{00} / N_{f}\right) /\left(4 C_{F}+N_{f}\right)$, where $T_{f}^{\prime 00} \equiv T_{f}^{00}-\sum_{f^{\prime}} T_{f^{\prime}}^{00} / N_{f}, T^{00} \equiv \sum_{f^{\prime}} T_{f^{\prime}}^{00}+T_{g}^{00}$, and $\tilde{T}^{00} \equiv 2 C_{F} \sum_{f^{\prime}} T_{f^{\prime}}^{00}-N_{f} T_{g}^{00} / 2$. Here, $T_{g}^{\mu \nu} \equiv-G_{a}^{\mu \alpha} G_{\alpha a}^{v}+g^{\mu \nu} G^{2} / 4$ is the gluon component of the traceless part of the energy-momentum tensor, $N_{f}$ the flavor number, and $C_{F} \equiv\left(N_{c}^{2}-1\right) /\left(2 N_{c}\right)$. A standard renormalization group (RG) analysis yields the following scaling properties [24]:

$$
T_{f}^{\prime 00}(\kappa)=\left[\frac{\ln \left(\kappa_{0} / \Lambda_{\mathrm{QCD}}\right)}{\ln \left(\kappa / \Lambda_{\mathrm{QCD}}\right)}\right]^{a^{\prime}} T_{f}^{\prime 00}\left(\kappa_{0}\right), \quad \tilde{T}^{00}(\kappa)=\left[\frac{\ln \left(\kappa_{0} / \Lambda_{\mathrm{QCD}}\right)}{\ln \left(\kappa / \Lambda_{\mathrm{QCD}}\right)}\right]^{\tilde{a}} \tilde{T}^{00}\left(\kappa_{0}\right),
$$

while $T^{00}$ is independent of $\kappa$. Here $\kappa$ and $\kappa_{0}$ are renormalization scales, $\Lambda_{\mathrm{QCD}}$ is the QCD scale parameter, $a^{\prime} \equiv 8 C_{F} /\left(3 b_{0}\right)$, and $\tilde{a} \equiv 2\left(4 C_{F}+N_{f}\right) /\left(3 b_{0}\right)$, where $b_{0} \equiv\left(11 N_{c}-2 N_{f}\right) / 3$, which appears in the expression $\alpha_{s}(\kappa)=2 \pi /\left[b_{0}\left(\ln \left(\kappa / \Lambda_{\mathrm{QCD}}\right)\right)\right]$. We see that, except for the $T^{00}$ term, all terms are suppressed logarithmically at large $\omega$. Thus, the resultant expression becomes

$$
\delta G^{R}(\omega)=e^{2} \sum_{f} q_{f}^{2} \frac{1}{\omega^{2}}\left[2 m_{f} \delta\left\langle\bar{\psi}_{f} \psi_{f}\right\rangle+\frac{1}{12} \delta\left\langle\frac{\alpha_{s}}{\pi} G^{2}\right\rangle+\frac{8}{3} \frac{\delta\left\langle T^{00}\right\rangle}{4 C_{F}+N_{f}}\right] .
$$

This vanishes at $\omega \rightarrow \infty$ and hence its contribution to Eq. (2) is zero. We note that, in $\omega \rightarrow \infty$ limit, which is relevant to the derivation of the sum rule, the asymptotic freedom of QCD guarantees that the above expression is exact. 
On the other hand, the IR limit is well described by hydrodynamics. At $|\mathbf{p}|=0$, it suffices to consider the constitutive relation for the system at rest, $\mathbf{j}=\sigma \mathbf{E}-\sigma \tau_{J} \partial_{t} \mathbf{E}+O\left(\partial^{2} E\right)$, since the conservation law of the current is trivial $\left(\partial_{t} j^{0}=-\nabla \cdot \mathbf{j}=0\right)$. Here $\sigma$ is the electrical conductivity, $\tau_{J}$ the second order transport coefficient for $\partial_{t} \mathbf{E}, \mathbf{E} \equiv-\nabla A^{0}-\partial_{0} \mathbf{A}$ the electric field, and $A^{\mu}$ the vector potential. We have dropped magnetic field dependent terms and the diffusion term from the constitutive relation, since they vanish in the $|\mathbf{p}|=0$ case. The linear response theory enables us to extract the retarded function through the relation,

$$
j_{\mu}(\omega)=-G_{\mu \nu}^{R}(\omega) A^{v}(\omega)
$$

which results in

$$
G^{R}(\omega)=i \omega \sigma\left(1+i \tau_{J} \omega\right)+O\left(\omega^{3}\right), \quad \rho(\omega)=\sigma \omega+O\left(\omega^{3}\right) .
$$

To get $\delta G^{R}$, we need to know $\left.G^{R}\right|_{T=0}$. Lorentz invariance guarantees the following form:

$$
\left.G^{R}(\omega)\right|_{T=0}=\omega^{2} G_{2}\left(\omega^{2}\right) .
$$

Here the real part of $G_{2}$ contains a UV divergence coming from the $T=0$ part, so the renormalization of the photon wave function $[24,25]$ is necessary, which implies $G_{2}(0)=0$. We note that $\sigma$ and $\tau_{J}$ in Eq. (7) need to be defined for the renormalized version of $G^{R}(\omega)$. Also the imaginary part of $G_{2}$ at small $\omega$ is zero because even the lightest vector meson (the $\rho$ meson) has non-zero mass and its spectral strength vanishes below the $\pi \pi$ threshold, so that the spectral weight around $\omega=0$ is zero. For these two reasons, we see that the left-hand side of Eq. (7) is actually equal to $\delta G^{R}$. This is not the case for the higher order terms that are of order $\omega^{4}$ or higher.

We also note that Eq. (7) is correct only in the large $N_{c}$ limit, in which the coupling effect among the hydro modes is negligible [26]. Beyond this limit, a nonanalytic term $\left(\sim \omega^{3 / 2}\right)$ appears in $\rho(\omega)$. Nevertheless, this does not affect the sum rules 1 and 2 we derive in this work. Applying the UV and IR results of Eqs. (5), (7), Eq. (2) becomes

$$
0=\int_{0}^{\infty} \frac{d \omega}{\omega} \delta \rho(\omega)
$$

This is the first sum rule (sum rule 1) to be discussed in this paper. We should mention here that this is the $|\mathbf{p}|=0$ version of the sum rule derived in Ref. [15].

\subsection{Sum Rule 2}

In a similar way (replacing $G^{R}$ with $\omega^{2} G^{R}$ in the derivation), we derive another sum rule which contains two more powers of $\omega$ in the integrand. In analogy to the derivation of sum rule 1, we get $\delta G_{0}^{R 2}-\delta G_{\infty}^{R 2}=2 \int_{0}^{\infty} d \omega \omega \delta \rho(\omega) / \pi$, where $\left.\delta G_{\infty}^{R 2} \equiv \omega^{2} \delta G^{R}(\omega)\right|_{\omega \rightarrow \infty}$ and $\left.\delta G_{0}^{R 2} \equiv \omega^{2} \delta G^{R}(\omega)\right|_{\omega \rightarrow 0}$. By using the UV/IR limits of $G^{R}$, Eqs. (5) and (7), we obtain

$$
\frac{2}{\pi} \int_{0}^{\infty} d \omega \omega \delta \rho(\omega)=-e^{2} \sum_{f} q_{f}^{2}\left[2 m_{f} \delta\left\langle\bar{\psi}_{f} \psi_{f}\right\rangle+\frac{1}{12} \delta\left\langle\frac{\alpha_{s}}{\pi} G^{2}\right\rangle+\frac{8}{3\left(4 C_{F}+N_{f}\right)} \delta\left\langle T^{00}\right\rangle\right]
$$




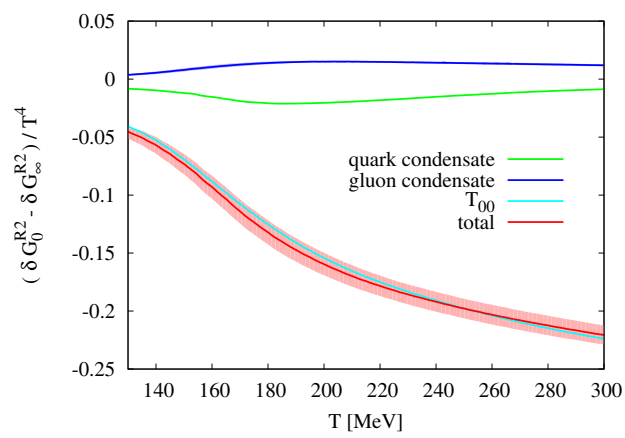

Figure 2. The right-hand sides of Eq. (10), divided by $T^{4}$ and shown as a function of temperature $T$. To extract the temperature dependence of the condensates, lattice QCD data provided in Ref. [27] were used. We used the value $e^{2}=0.092$ for the plots.

This is the second sum rule (sum rule 2$)^{1}$ we discuss in this work.

It should be emphasized here that the condensates appearing on the right-hand side of this sum rule are static quantities, that can be evaluated non-perturbatively from lattice QCD. The gluon condensate can be computed by using the relation at leading order in $\alpha_{s}$,

$$
e-3 p=\sum_{f} m_{f} \delta\left\langle\bar{\psi}_{f} \psi_{f}\right\rangle-\frac{11 N_{c}-2 N_{f}}{24} \delta\left\langle\frac{\alpha_{s}}{\pi} G^{2}\right\rangle .
$$

We note that though the sum rule (10) is exact, the evaluation of the gluon condensate using the expression above is valid only perturbatively. In this study, we take the chiral condensate, energy and pressure from a recent $N_{f}=2+1$ lattice calculation by the HotQCD Collaboration [27]. To understand the behavior of the different terms on the right-hand side of Eq. (10), they are shown in Fig. 2 as a function of temperature. It is seen in this figure that the quark and gluon condensate terms are relatively small, comparable in magnitude and have opposite signs. Their contributions therefore cancel to a large degree, so that the right-hand side of Eq. (10) is almost completely determined by the dominant $\delta\left\langle T^{00}\right\rangle$ term. Because this term does not depend on quark flavor, the decomposition of Eq. (10) into its flavor components is determined simply by the quark charges $q_{f}$, meaning that the $u$-quark contribution is about a factor of four larger than those of the $d$ and $s$-quarks.

\subsection{Sum Rule 3}

In the sum rule to be discussed in this subsection, the integrand of sum rule 1 is in essence divided by $\omega^{2}$. To avoid potential IR divergences, the derivation however has to be carried out with some care.

\footnotetext{
${ }^{1}$ We note that this sum rule in the case of $N_{f}=1$ and $N_{c}=3$ was derived in Ref. [9]. However, the coefficient of $T^{00}$ in this reference is not the correct one (Eq. (10)), but is equal to that in the expression where the effect of the mixing/rescaling of the energy-momentum tensor is neglected.
} 
Equation (1) can be written as

$$
\begin{aligned}
\delta G^{R}(i \omega)-\delta G_{\infty}^{R} & =\frac{1}{2 \pi} \int_{-\infty}^{\infty} d \omega^{\prime} \frac{1}{\omega^{\prime 2}+\omega^{2}}\left(\omega^{\prime} \delta \rho\left(\omega^{\prime}\right)+\omega \operatorname{Re}\left[\delta G^{R}\left(\omega^{\prime}\right)-\delta G_{\infty}^{R}\right]\right) \\
& =\frac{1}{\pi} \int_{-\infty}^{\infty} d \omega^{\prime} \frac{\omega^{\prime} \delta \rho\left(\omega^{\prime}\right)}{\omega^{\prime 2}+\omega^{2}},
\end{aligned}
$$

where in the second line we have used the property that the contributions from the first and the second terms are equal, which can be shown by evaluating the right-hand side of Eq. (1) using the residue theorem with the contour closing in the lower half plane. Subtracting Eq. (2) and $-\sigma \omega$ from this expression and using Eq. (7) on the left-hand side, we get

$$
-\sigma \tau_{J}=\frac{2}{\pi} \int_{0}^{\infty} \frac{d \omega}{\omega^{3}}[\delta \rho(\omega)-\sigma \omega],
$$

in which the $-\sigma \omega$ term in the integrand is included to remove the IR singularity. This is the third sum rule (sum rule 3 ) we have derived in this paper.

\section{Application to Lattice QCD}

Let us demonstrate that the sum rules we have derived can be used to give constraints to the spectral ansatz used in fits to lattice QCD data. As a first trial, we consider the simple ${ }^{2}$ ansatz introduced in Ref. [13] (all quantities proportional to $\rho(\omega)$ or $G^{R}$ in this work are multiplied by a factor of $1 / 6$ compared to the corresponding expressions in Ref. [13]),

$$
\rho(\omega)=C_{\mathrm{em}}\left[c_{B W} \rho_{\text {peak }}(\omega)+(1+k) \rho_{\text {cont }}(\omega)\right]
$$

where

$$
\begin{aligned}
\rho_{\text {peak }}(\omega) & \equiv \frac{1}{3} \frac{\omega \Gamma / 2}{\omega^{2}+(\Gamma / 2)^{2}}, \\
\rho_{\text {cont }}(\omega) & \equiv \frac{\omega^{2}}{4 \pi}\left(1-2 n_{F}\left(\frac{\omega}{2}\right)\right),
\end{aligned}
$$

correspond to the transport peak and the continuum in the weak coupling limit. Here $n_{F}(|\mathbf{k}|) \equiv$ $[\exp (|\mathbf{k}| / T)+1]^{-1}$ is the distribution function at equilibrium and $C_{\mathrm{em}} \equiv e^{2} \sum_{f} q_{f}^{2}$. We note that, $\delta \rho(\omega)$ can be obtained by subtracting $\rho_{T=0}(\omega)$. Data for this function can be obtained from the experimental ( $e^{+} e^{-} \rightarrow$ hadrons) cross section (see for instance the compilation of data given in the particle data group [28]), or from zero temperature lattice calculations. In this paper, we will however for simplicity confine ourselves to the averaged form $C_{\mathrm{em}} \omega^{2}(1+k) /(4 \pi)^{3}$. Equation (14) contains three parameters $\left(c_{B W}, \Gamma, k\right)$ that need to be determined by fitting the data. Sum rule 1 of Eq. (9) provides a constraint on these parameters:

$$
c_{B W}=(1+k) T^{2} .
$$

\footnotetext{
${ }^{2}$ A more complicated ansatz, which also contains information on vacuum bound states, was introduced in Refs. [14, 16]. In these works, the sum rule of Eq. (9) was furthermore used to constrain the parameters appearing in their ansatz.

${ }^{3}$ This ansatz could be improved by taking into account the lowest few resonances of the spectrum, similar to Refs. [14, 16], or by making direct use of the $\left(e^{+} e^{-} \rightarrow\right.$ hadrons) cross section data.
} 


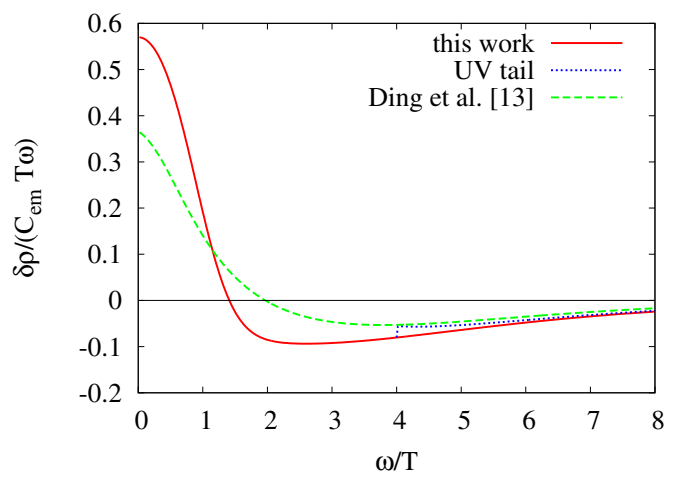

Figure 3. Ansatz A (red solid line), ansatz B (blue dotted line), and the ansatz used in Ref. [13] (green dashed line) as functions of $\omega$. The unit of the vertical axis is $C_{e m} T \omega$ while that of the horizontal axis is $T$.

This constraint may be used to reduce the number of fitting parameters in the ansatz. Here, we simply check whether the values of the parameters obtained from the fit [13] satisfy the sum rule. The fitted values at $T=1.45 T_{c}$ are $k \simeq 0.047, \Gamma \simeq 2.2 T, c_{B W} \simeq 1.2 T^{2}$, which give $1.2 T^{2}$ on the left-hand side of Eq. (17) while $1.0 T^{2}$ is obtained on the right-hand side. We see that, even though the agreement is not perfect, the fit satisfies the constraint with reasonable precision.

Nevertheless, the ansatz Eq. (14) can not be applied to the other two sum rules, Eqs. (10) and (13), because it would cause a UV divergence in sum rule 2 and an IR divergences in sum rule 3. Therefore, to construct a spectral function that can satisfy all three sum rules, an improved parametrization is necessary. We hence propose the following ansatz (ansatz A):

$$
\rho(\omega)=C_{\mathrm{em}}\left[c_{B W} \rho_{\text {peak }}(\omega)[1-A(\omega)]+A(\omega)(1+k) \rho_{\mathrm{cont}}(\omega)\right],
$$

where $A(\omega) \equiv \tanh \left(\omega^{2} / \Delta^{2}\right)$. As one can easily check, the cutoff function $A(\omega)$ removes all IR and UV divergences in Eqs. (10) and (13).

The values of $\Gamma, c_{B W}, k$, and $\Delta$ should be determined from lattice data. To demonstrate that this functional form is feasible, we have performed a simple trial analysis, making use of the Euclidean vector correlator and second thermal moment data provided in Ref. [13] for $T=1.45 T_{c}$. These data were also used to fit the ansatz of Eq. (14), as explained above. The Euclidean vector correlator is given in terms of the spectral function as

$$
G^{\mathrm{E}}(\tau, T)=\int_{0}^{\infty} \frac{d \omega}{2 \pi} \rho(\omega) \frac{\cosh [\omega(\tau-1 / 2 T)]}{\sinh (\omega / 2 T)},
$$

with Euclidian time $\tau$, while the second thermal moment is defined as

$$
G^{(2)}(T)=\frac{1}{2} \int_{0}^{\infty} \frac{d \omega}{2 \pi}\left(\frac{\omega}{T}\right)^{2} \frac{\rho(\omega)}{\sinh (\omega / 2 T)} .
$$




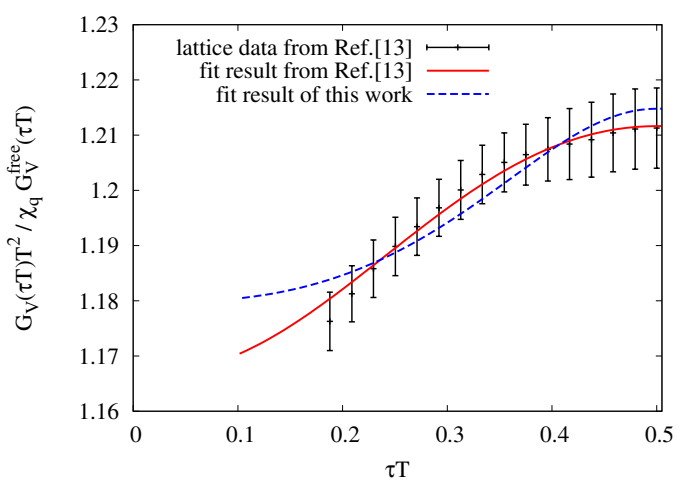

Figure 4. Lattice data for the Euclidean vector correlator, adapted from Ref. [13] (black points), the fit result using Eqs. (14-16) (red solid line) and the fit result using the improved functional form of Eq. (18) (blue dashed line).

In Ref. [13] the latter quantity was given relative to its free counterpart:

$$
\frac{G^{(2)}(T)}{G_{\text {free }}^{(2)}(T)}=1.067 \pm 0.012 \quad\left(T=1.45 T_{c}\right) .
$$

Here, the free second thermal moment can be computed analytically and is in our conventions given as

$$
G_{\text {free }}^{(2)}(T)=\frac{14 \pi^{2}}{15} T^{3}
$$

We moreover employ the sum rule 1 of Eq. (9) to constrain our fit, as it was done in Ref. [14, 16]. Specifically, the constraints of the second thermal moment [Eqs. (20) and (21)] and sum rule 1 allow us to determine $c_{B W}$ and $k$ and therefore to reduce the number of undetermined parameters to two ( $\Gamma$ and $\Delta$ ), which are then fitted to the Euclidean vector correlator data. In this fit, we do not only use the central value of Eq. (21), but probe the whole range to look for the value that gives the smallest overall $\chi^{2}$. Following this procedure, we have found that the best fit is obtained for very large values of $\Gamma$, with values of $\Delta / T$ of the order of one. This means that the transport peak at low energy is not generated by the Lorentzian of Eq. (15), but by the function $1-A(\omega)$, with the width $2 \Delta$. As will be shown in Fig. 3, these two functional forms are quite alike and share many qualitative features. Quantitatively, our best fit is obtained for,

$$
\begin{aligned}
k & =0.058, \quad 2 c_{B W} /(T \Gamma)=1.7, \\
\Gamma / T & =\text { infinity, } \quad \Delta / T=1.2,
\end{aligned}
$$

which gives a $\chi^{2} /$ d.o.f of 0.53 . The respective (vacuum subtracted) spectral function is shown in Fig. 3, together with the fit result of Ref. [13], for which Eq. (14) was used. To give the reader a better idea on the quality of the fit, we show in Fig. 4 the Euclidean vector correlator lattice data with our fitted curve. For comparison, we also plot the curve corresponding to the fit performed in Ref. [13] 
with Eqs. (14-16). $G_{\mathrm{V}}(\tau, T)$, which is used in Fig. 4 is defined as $G_{\mathrm{V}}(\tau, T)=G^{\mathrm{E}}(\tau, T)-\chi_{q} T / 6$. For the quark number susceptibility $\chi_{q}$ we employ the value provided in Ref. [13]: $\chi_{q} / T^{2}=0.897$. Furthermore, $G_{\mathrm{V}}^{\text {free }}(\tau, T)$ is related to the free Euclidean vector correlator and can be given analytically as

$$
G_{\mathrm{V}}^{\mathrm{free}}(\tau, T)=T^{3}\left[\pi(1-2 \tau T) \frac{1+\cos ^{2}(2 \pi \tau T)}{\sin ^{3}(2 \pi \tau T)}+2 \frac{\cos (2 \pi \tau T)}{\sin ^{2}(2 \pi \tau T)}\right] .
$$

It is seen in Fig. 4 that the fit of Ref. [13] generally agrees better with the central values of the lattice data points. Their errors are however too large to discriminate the two fits. Reduced errors and more data points at smaller $\tau T$ values will likely improve this situation and impose tougher constraints on the various functional forms used to parametrized the spectral function.

Having the fitted and well behaved spectral function of Eq. (18) at hand, we can now proceed to compute various quantities of interest. First of all, one can easily extract the electrical conductivity as

$$
\begin{aligned}
\frac{\sigma}{T} & =\lim _{\omega \rightarrow 0} \frac{\rho(\omega)}{\omega T}=C_{\mathrm{em}} \frac{2 c_{B W}}{3 T \Gamma} \\
& =0.57 \times C_{\mathrm{em}},
\end{aligned}
$$

which is about $50 \%$ larger than the value reported in [13].

As a last point, we next discuss the application of sum rule 3 given in Eq. (13). At first, let us clarify the definition of the parameter appearing in the left-hand side, $\tau_{J}$. It is expressed in terms of the retarded Green function as, $\tau_{J} \equiv-G^{R \prime \prime}(\omega=0) /(2 \sigma)$ as can be seen from Eq. (7). $\tau_{J}$ therefore does not explicitly appear in the spectral function since it corresponds to the real part of $G^{R}$. As the transport coefficient $\tau_{J}$ is furthermore at present not known, this sum rule can not be used as an additional fitting constraint. If the spectral function is however already determined from other sources, Eq. (13) can be used to estimate $\tau_{J}$. Using Eqs. (18-25), we get

$$
\tau_{J}=0.067 C_{\mathrm{em}} / T \quad\left(T=1.45 T_{c}\right) .
$$

To our knowledge, this is the first time that this transport coefficient has been determined nonperturbatively. Note that the above number is a quenched QCD estimate, as we have made use of quenched lattice data to fix the spectral function. We should furthermore mention here that, all the ansätze used in this section do not take into account the large $N_{c}$ suppressed nonanalytic behavior at small $\omega$ (which seems to be challenging to see in current lattice QCD analysis) caused by hydro mode coupling. It is therefore consistent to use sum rule 3 (13), which does not consider this effect as well.

\section{ACKNOWLEDGMENTS}

D.S. is supported by the Alexander von Humboldt Foundation.

\section{References}

[1] L. D. McLerran and T. Toimela, Phys. Rev. D 31, 545 (1985).

[2] R. Baier, B. Pire and D. Schiff, Phys. Rev. D 38, 2814 (1988); M. Laine, JHEP 1311, 120 (2013) [arXiv:1310.0164 [hep-ph]].

[3] S. Caron-Huot, P. Kovtun, G. D. Moore, A. Starinets and L. G. Yaffe, JHEP 0612, 015 (2006) [hep-th/0607237]. 
[4] C. Gale, Y. Hidaka, S. Jeon, S. Lin, J.-F. Paquet, R. D. Pisarski, D. Satow and V. V. Skokov et al., Phys. Rev. Lett. 114, 072301 (2015) [arXiv:1409.4778 [hep-ph]]; Y. Hidaka, S. Lin, R. D. Pisarski and D. Satow, JHEP 1510, 005 (2015) [arXiv:1504.01770 [hep-ph]]; D. Satow and W. Weise, Phys. Rev. D 92, 056001 (2015) [arXiv:1505.03869 [hep-ph]]; C. A. Islam, S. Majumder, N. Haque and M. G. Mustafa, JHEP 1502, 011 (2015) [arXiv:1411.6407 [hep-ph]].

[5] G. Chanfray, R. Rapp and J. Wambach, Phys. Rev. Lett. 76, 368 (1996) [hep-ph/9508353].

[6] F. Klingl, N. Kaiser and W. Weise, Nucl. Phys. A 624, 527 (1997) [hep-ph/9704398].

[7] P. Gubler and W. Weise, Phys. Lett. B 751, 396 (2015) [arXiv:1507.03769 [hep-ph]]; P. M. Hohler and R. Rapp, Nucl. Phys. A 892, 58 (2012) [arXiv:1204.6309 [hep-ph]]; Phys. Lett. B 731, 103 (2014) [arXiv:1311.2921 [hep-ph]]; C. A. Dominguez, M. Loewe, J. C. Rojas and Y. Zhang, Phys. Rev. D 81, 014007 (2010) [arXiv:0908.2709 [hep-ph]];

[8] J. I. Kapusta and E. V. Shuryak, Phys. Rev. D 49, 4694 (1994) doi:10.1103/PhysRevD.49.4694 [hep-ph/9312245].

[9] S. Z. Huang and M. Lissia, Phys. Lett. B 348, 571 (1995) [hep-ph/9404275]; Phys. Rev. D 52, 1134 (1995) [hep-ph/9412246].

[10] S. Zschocke, O. P. Pavlenko and B. Kampfer, Eur. Phys. J. A 15, 529 (2002) [nucl-th/0205057].

[11] G. Aarts, C. Allton, J. Foley, S. Hands and S. Kim, Phys. Rev. Lett. 99, 022002 (2007) [hep-lat/0703008 [HEP-LAT]]; A. Amato, G. Aarts, C. Allton, P. Giudice, S. Hands and J. I. Skullerud, Phys. Rev. Lett. 111, 172001 (2013) [arXiv:1307.6763 [hep-lat]]; JHEP 1502, 186 (2015) [arXiv:1412.6411 [hep-lat]].

[12] F. Karsch, E. Laermann, P. Petreczky, S. Stickan and I. Wetzorke, Phys. Lett. B 530, 147 (2002) [hep-lat/0110208].

[13] H. -T. Ding, A. Francis, O. Kaczmarek, F. Karsch, E. Laermann and W. Soeldner, Phys. Rev. D 83, 034504 (2011) [arXiv:1012.4963 [hep-lat]].

[14] B. B. Brandt, A. Francis, B. Jaeger and H. B. Meyer, arXiv:1512.07249 [hep-lat].

[15] D. Bernecker and H. B. Meyer, Eur. Phys. J. A 47, 148 (2011) [arXiv:1107.4388 [hep-lat]].

[16] B. B. Brandt, A. Francis, H. B. Meyer and H. Wittig, JHEP 1303, 100 (2013) [arXiv:1212.4200 [hep-lat]].

[17] B. B. Brandt, A. Francis, H. B. Meyer and D. Robaina, Phys. Rev. D 90, no. 5, 054509 (2014) [arXiv:1406.5602 [hep-lat]].

[18] S. Gupta, Phys. Lett. B 597, 57 (2004) [hep-lat/0301006].

[19] Y. Burnier and M. Laine, Eur. Phys. J. C 72, 1902 (2012) [arXiv:1201.1994 [hep-lat]].

[20] P. Romatschke and D. T. Son, Phys. Rev. D 80, 065021 (2009) [arXiv:0903.3946 [hep-ph]].

[21] P. Gubler and D. Satow, arXiv:1602.08265 [hep-ph].

[22] S. Caron-Huot, Phys. Rev. D 79, 125009 (2009) [arXiv:0903.3958 [hep-ph]].

[23] M. A. Shifman, A. I. Vainshtein and V. I. Zakharov, Nucl. Phys. B 147, 448 (1979). doi:10.1016/0550-3213(79)90023-3

[24] M. E. Peskin and D. V. Schroeder, Reading, USA: Addison-Wesley (1995) 842 p

[25] G. S. Bali, F. Bruckmann, G. Endrödi, S. D. Katz and A. Schäfer, JHEP 1408, 177 (2014) doi:10.1007/JHEP08(2014)177 [arXiv:1406.0269 [hep-lat]].

[26] P. Kovtun and L. G. Yaffe, Phys. Rev. D 68, 025007 (2003) [hep-th/0303010].

[27] A. Bazavov et al. [HotQCD Collaboration], Phys. Rev. D 90, no. 9, 094503 (2014) doi:10.1103/PhysRevD.90.094503 [arXiv:1407.6387 [hep-lat]].

[28] K.A. Olive et al. (Particle Data Group), Chin. Phys. C 38, 090001 (2014). 